(c) Canadian Mathematical Society 2021. This is an Open Access article, distributed under the terms of the Creative Commons Attribution licence (http://creativecommons.org/licenses/ by/4.0/), which permits unrestricted re-use, distribution, and reproduction in any medium, provided the original work is properly cited.

\title{
Log-concavity and log-convexity of moments of averages of i.i.d. random variables
}

\author{
Philip Lamkin and Tomasz Tkocz
}

Abstract. We show that the sequence of moments of order less than 1 of averages of i.i.d. positive random variables is log-concave. For moments of order at least 1, we conjecture that the sequence is log-convex and show that this holds eventually for integer moments (after neglecting the first $p^{2}$ terms of the sequence).

\section{Introduction and results}

Suppose $X_{1}, X_{2}, \ldots$ are i.i.d. copies of a positive random variable and $f$ is a nonnegative function. This article is concerned with certain combinatorial properties of the sequence

$$
a_{n}=\mathbb{E} f\left(\frac{X_{1}+\cdots+X_{n}}{n}\right), \quad n=1,2, \ldots
$$

For instance, $f(x)=x^{p}$ is a fairly natural choice leading to the sequence of moments of averages of the $X_{i}$. Because we have the identity

$$
\sum_{i=1}^{n+1} x_{i}=\sum_{i=1}^{n+1} \frac{\sum_{j: j \neq i} x_{j}}{n},
$$

we conclude that the sequence $\left(a_{n}\right)_{n=1}^{\infty}$ is nonincreasing when $f$ is convex. What about inequalities involving more than two terms?

Such inequalities have been studied to some extent. One fairly general result is due to Boland, Proschan, and Tong from [1] (with applications in reliability theory). It asserts in particular that for $n=2,3, \ldots$,

$$
\mathbb{E} \phi\left(X_{1}+\cdots+X_{n}, X_{n+1}+\cdots+X_{2 n}\right) \leq \mathbb{E} \phi\left(X_{1}+\cdots+X_{n-1}, X_{n}+\cdots+X_{2 n}\right)
$$

for a symmetric (invariant under permuting coordinates) continuous random vector $X=\left(X_{1}, \ldots, X_{2 n}\right)$ with nonnegative components and a symmetric convex function $\phi:[0,+\infty)^{2} \rightarrow \mathbb{R}$.

Received by the editors April 21, 2020; revised April 22, 2021, accepted April 23, 2021.

Published online on Cambridge Core May 3, 2021.

This research was supported in part by the Collaboration Grants from the Simons Foundation.

AMS subject classification: 05A20, 60E15, 26D15.

Keywords: Log-convexity, log-concavity, moment comparison, sums of independent random variables. 
We obtain a satisfactory answer to a natural question of log-convexity/concavity of sequences $\left(a_{n}\right)$ for completely monotone functions, also providing insights into the case of power functions.

Recall that a nonnegative sequence $\left(x_{n}\right)_{n=1}^{\infty}$ supported on a set of contiguous integers is called log-convex (resp. log-concave) if $x_{n}^{2} \leq x_{n-1} x_{n+1}\left(\right.$ resp. $\left.x_{n}^{2} \geq x_{n-1} x_{n+1}\right)$ for all $n \geq 2$ (for background on log-convex/concave sequences, see, for instance, [8, $12])$. One of the crucial properties of log-convex sequences is that log-convexity is preserved by taking sums (which follows from the Cauchy-Schwarz inequality, see, for instance, [8]). Recall that an infinitely differentiable function $f:(0, \infty) \rightarrow(0, \infty)$ is called completely monotone if we have $(-1)^{n} f^{(n)}(x) \geq 0$ for all positive $x$ and $n=1,2, \ldots$; equivalently, by Bernstein's theorem (see, for instance, [7]), the function $f$ is the Laplace transform of a nonnegative Borel measure $\mu$ on $[0,+\infty)$, that is,

$$
f(x)=\int_{0}^{\infty} e^{-t x} \mathrm{~d} \mu(t)
$$

For example, when $p<0$, the function $f(x)=x^{p}$ is completely monotone. Such integral representations are at the heart of our first two results.

Theorem 1 Let $f:(0, \infty) \rightarrow(0, \infty)$ be a completely monotone function. Let $X_{1}, X_{2}, \ldots$ be i.i.d. positive random variables. Then, the sequence $\left(a_{n}\right)_{n=1}^{\infty}$ defined by (1) is log-convex.

Theorem 2 Let $f:[0, \infty) \rightarrow[0, \infty)$ be such that $f(0)=0$ and its derivative $f^{\prime}$ is completely monotone. Let $X_{1}, X_{2}, \ldots$ be i.i.d. nonnegative random variables. Then, the sequence $\left(a_{n}\right)_{n=1}^{\infty}$ defined by (1) is log-concave.

In particular, applying these to the functions $f(x)=x^{p}$ with $p<0$ and $0<p<1$, respectively, we obtain the following corollary.

Corollary 3 Let $X_{1}, X_{2}, \ldots$ be i.i.d. positive random variables. The sequence

$$
b_{n}=\mathbb{E}\left(\frac{X_{1}+\cdots+X_{n}}{n}\right)^{p}, \quad n=1,2, \ldots
$$

is log-convex when $p<0$ and log-concave when $0<p<1$.

For $p>1$, we pose the following conjecture.

Conjecture 1 Let $p>1$. Let $X_{1}, X_{2}, \ldots$ be i.i.d. nonnegative random variables. Then, the sequence $\left(b_{n}\right)$ defined in (4) is log-convex.

We offer a partial result supporting this conjecture.

Theorem 4 Let $X_{1}, X_{2}, \ldots$ be i.i.d. nonnegative random variables, let $p$ be a positive integer, and let $b_{n}$ be defined by (4). Then, for every $n \geq p^{2}$, we have $b_{n}^{2} \leq b_{n-1} b_{n+1}$. 
Remark 5 When $p=2$, we have $b_{n}=\frac{n \mathbb{E} X_{1}^{2}+n(n-1)\left(\mathbb{E} X_{1}\right)^{2}}{n^{2}}=\left(\mathbb{E} X_{1}\right)^{2}+n^{-1} \operatorname{Var}\left(X_{1}\right)$, which is clearly a log-convex sequence (as a sum of two log-convex sequences). The following argument for $p=3$ was kindly communicated to us by Krzysztof Oleszkiewicz: when $p=3$, we can write

$$
b_{n}=\left(\mathbb{E} X_{1}\right)^{3}+\left(\mathbb{E} X_{1}^{3}+\left(\mathbb{E} X_{1}\right)^{3}-2\left(\mathbb{E} X_{1}^{2}\right)\left(\mathbb{E} X_{1}\right)\right) n^{-2}+\left(\mathbb{E} X_{1}\right) \operatorname{Var}\left(X_{1}\right)\left(3 n^{-1}-n^{-2}\right) .
$$

The sequences $\left(n^{-2}\right)$ and $\left(3 n^{-1}-n^{-2}\right)$ are log-convex. By the Cauchy-Schwarz inequality, the factor at $n^{-2}$ is nonnegative,

$$
\mathbb{E} X_{1}^{2} \leq \sqrt{\mathbb{E} X_{1}^{3}} \sqrt{\mathbb{E} X_{1}} \leq \frac{\mathbb{E} X_{1}^{3}}{2 \mathbb{E} X_{1}}+\frac{\left(\mathbb{E} X_{1}\right)^{2}}{2},
$$

so again $\left(b_{n}\right)$ is log-convex as a sum of three log-convex sequences. It remains elusive how to group terms and proceed along these lines in general. Our proof of Theorem 4 relies on this idea, but uses a straightforward way of rearranging terms.

Remark 6 It would be tempting to use the aforementioned result of Boland et al. with $\phi(x, y)=(x y)^{p}$ to resolve Conjecture 1 . However, this function is neither convex nor concave on $(0,+\infty)^{2}$ for $p>\frac{1}{2}$. For $0<p<\frac{1}{2}$, the function is concave and (2) yields $\left(b_{n} n^{p}\right)^{2} \geq b_{n-1}(n-1)^{p} b_{n+1}(n+1)^{p}, n \geq 2$, equivalently, $b_{n}^{2} \geq$ $\left(\frac{n^{2}-1}{n^{2}}\right)^{p} b_{n-1} b_{n+1}$. Corollary 3 improves on this by removing the factor $\left(\frac{n^{2}-1}{n^{2}}\right)^{p}<1$. For $p<0, \phi$ is convex, so (2) gives $b_{n}^{2} \leq\left(\frac{n^{2}-1}{n^{2}}\right)^{p} b_{n-1} b_{n+1}$ and Corollary 3 removes the factor $\left(\frac{n^{2}-1}{n^{2}}\right)^{p}>1$.

Concluding this introduction, it is of significant interest to study the log-behavior of various sequences, particularly those emerging from algebraic, combinatorial, or geometric structures, which has involved and prompted the development of many deep and interesting methods, often useful beyond the original problems (see, e.g., [3-6, 10-13]). We propose to consider sequences of moments of averages of i.i.d. random variables arising naturally in probabilistic limit theorems. For moments of order less than 1, we employ an analytical approach exploiting integral representations for power functions. For moments of order higher than 1, our Conjecture 1, besides refining the monotonicity property of the sequence $\left(b_{n}\right)$ (resulting from convexity), would furnish new examples of log-convex sequences. For instance, neither does it seem trivial, nor handled by known techniques, to determine whether the sequence, obtained by taking the Bernoulli distribution with parameter $\theta \in(0,1)$, $b_{n}=\sum_{k=0}^{n}\left(\begin{array}{l}n \\ k\end{array}\right)\left(\frac{k}{n}\right)^{p} \theta^{k}(1-\theta)^{n-k}$ is log-convex. In the case of integral $p$, we have $b_{n}=\sum_{k=0}^{p} S(p, k) \frac{n !}{(n-k) ! n^{p}} \theta^{k}$, where $S(p, k)$ is the Stirling number of the second kind.

The rest of this paper is occupied with the proofs of Theorems 1,2, and 4 (in their order of statement), and then we conclude with additional remarks and conjectures. 


\section{Proofs}

\subsection{Proof of Theorem 1}

Suppose that $f$ is completely monotone. Using (3) and independence, we have

$$
a_{n}=\mathbb{E} f\left(\frac{X_{1}+\cdots+X_{n}}{n}\right)=\int_{0}^{\infty}\left[\mathbb{E} e^{-t X_{1} / n}\right]^{n} \mathrm{~d} \mu(t) .
$$

Let $u_{n}(t)=\left[\mathbb{E} e^{-t X_{1} / n}\right]^{n}$. It suffices to show that for every positive $t$, the sequence $\left(u_{n}(t)\right)$ is log-convex (because sums/integrals of log-convex sequences are logconvex: the Cauchy-Schwarz inequality applied to the measure $\mu$ yields

$$
\left(\int \sqrt{u_{n-1}(t) u_{n+1}(t)} \mathrm{d} \mu(t)\right)^{2} \leq\left(\int u_{n-1}(t) \mathrm{d} \mu(t)\right)\left(\int u_{n+1}(t) \mathrm{d} \mu(t)\right),
$$

which combined with $u_{n}(t) \leq \sqrt{u_{n-1}(t) u_{n+1}(t)}$, gives $\left.a_{n}^{2} \leq a_{n-1} a_{n+1}\right)$. The logconvexity of $\left(u_{n}(t)\right)$ follows from Hölder's inequality,

$$
\mathbb{E} e^{-t X_{1} / n}=\mathbb{E} e^{-\frac{n-1}{2 n} \frac{t X_{1}}{n-1}} e^{-\frac{n+1}{2 n} \frac{t X_{1}}{n+1}} \leq\left(\mathbb{E} e^{-\frac{t X_{1}}{n-1}}\right)^{\frac{n-1}{2 n}}\left(\mathbb{E} e^{-\frac{t X_{1}}{n+1}}\right)^{\frac{n+1}{2 n}},
$$

which finishes the proof.

\subsection{Proof of Theorem 2}

Suppose now that $f(0)=0$ and $f^{\prime}$ is completely monotone, say $f^{\prime}(x)=\int_{0}^{\infty} e^{-t x} \mathrm{~d} \mu(t)$ for some nonnegative Borel measure $\mu$ on $(0, \infty)$ (by (3)). Introducing a new measure $\mathrm{d} v(t)=\frac{1}{t} \mathrm{~d} \mu(t)$, we can write

$$
f(y)=f(y)-f(0)=\int_{0}^{y} f^{\prime}(x) \mathrm{d} x=\int_{0}^{\infty} \int_{0}^{\infty} t e^{-t x} 1_{\{0<x<y\}} \mathrm{d} x \mathrm{~d} v(t) .
$$

Integrating against $\mathrm{d} x$ gives

$$
f(y)=\int_{0}^{\infty}\left[1-e^{-t y}\right] \mathrm{d} v(t) .
$$

Let $F$ be the Laplace transform of $X_{1}$, that is,

$$
F(t)=\mathbb{E} e^{-t X_{1}}, \quad t>0 .
$$

Then,

$$
\mathbb{E} f\left(\frac{X_{1}+\cdots+X_{n}}{n}\right)=\int_{0}^{\infty}\left[1-F(t / n)^{n}\right] \mathrm{d} v(t)=\int_{0}^{\infty} G(n, t) \mathrm{d} v(t),
$$

where, to shorten the notation, we introduce the following nonnegative function:

$$
G(\alpha, t)=1-F(t / \alpha)^{\alpha}, \quad \alpha, t>0 .
$$

To show the inequality

$$
\left[\mathbb{E} f\left(\frac{X_{1}+\cdots+X_{n}}{n}\right)\right]^{2} \geq \mathbb{E} f\left(\frac{X_{1}+\cdots+X_{n-1}}{n-1}\right) \cdot \mathbb{E} f\left(\frac{X_{1}+\cdots+X_{n+1}}{n+1}\right),
$$


it suffices to show that pointwise

$$
G(n, s) G(n, t) \geq \frac{1}{2} G(n-1, s) G(n+1, t)+\frac{1}{2} G(n+1, s) G(n-1, t),
$$

for all $s, t>0$. This follows from two properties of the function $G$ :

(1) for every fixed $t>0$, the function $\alpha \mapsto G(\alpha, t)$ is nondecreasing,

(2) the function $G(\alpha, t)$ is concave on $(0, \infty) \times(0, \infty)$.

Indeed, by (2), we have

$$
G(n, s) G(n, t) \geq \frac{G(n-1, s)+G(n+1, s)}{2} \cdot \frac{G(n-1, t)+G(n+1, t)}{2}
$$

(in fact, we only use concavity in the first argument). It thus suffices to prove that

$$
\begin{aligned}
& G(n-1, s) G(n-1, t)+G(n+1, s) G(n+1, t) \\
& \quad-G(n-1, s) G(n+1, t)-G(n+1, s) G(n-1, t) \\
& =[G(n-1, s)-G(n+1, s)] \cdot[G(n-1, t)-G(n+1, t)]
\end{aligned}
$$

is nonnegative, which follows by (1).

It remains to prove (1) and (2). To prove the former, notice that $F(t / \alpha)^{\alpha}=$ $\left(\mathbb{E} e^{-t X / \alpha}\right)^{\alpha}=\left\|e^{-t X}\right\|_{1 / \alpha}$ is the $L_{1 / \alpha}$-norm of $e^{-t X}$. By convexity, for an arbitrary random variable $Z, p \mapsto\left(\mathbb{E}|Z|^{p}\right)^{1 / p}=\|Z\|_{p}$ is nondecreasing, so $F(t / \alpha)^{\alpha}=\left\|e^{-t X}\right\|_{1 / \alpha}$ is nonincreasing and thus $G(\alpha, t)=1-F(t / \alpha)^{\alpha}$ is nondecreasing. To prove the latter, notice that by Hölder's inequality the function $t \mapsto \log F(t)$ is convex,

$$
F(\lambda s+(1-\lambda) t)=\mathbb{E}\left[\left(e^{-s X}\right)^{\lambda}\left(e^{-t X}\right)^{1-\lambda}\right] \leq\left(\mathbb{E} e^{-s X}\right)^{\lambda}\left(\mathbb{E} e^{-t X}\right)^{1-\lambda}=F(s)^{\lambda} F(t)^{1-\lambda} .
$$

Therefore, its perspective function $H(\alpha, t)=\alpha \log F(t / \alpha)$ is convex (see, e.g., Chapter 3.2.6 in [2]), which implies that $F(t / \alpha)^{\alpha}=e^{H(\alpha, t)}$ is also convex.

\subsection{Proof of Theorem 4}

We recall a standard combinatorial formula: first, by the multinomial theorem and independence, we have

$$
\mathbb{E}\left(X_{1}+\cdots+X_{n}\right)^{p}=\sum \frac{p !}{p_{1} ! \cdots p_{n} !} \mathbb{E}\left(X_{1}^{p_{1}} \cdot \ldots \cdot X_{n}^{p_{n}}\right)=\sum \frac{p !}{p_{1} ! \cdots p_{n} !} \mu_{p_{1}} \cdot \ldots \cdot \mu_{p_{n}},
$$

where the sum is over all sequences $\left(p_{1}, \ldots, p_{n}\right)$ of nonnegative integers such that $p_{1}+\cdots+p_{n}=p$ and we denote $\mu_{k}=\mathbb{E} X_{1}^{k}, k \geq 0$. Now, we partition the summation according to the number $m$ of positive terms in the sequence $\left(p_{1}, \ldots, p_{n}\right)$. Let $Q_{m}$ be the set of integer partitions of $p$ into exactly $m$ (nonempty) parts, that is, the set of $m$-element multisets $q=\left\{q_{1}, \ldots, q_{m}\right\}$ with positive integers $q_{j}$ summing to $p, q_{1}+$ $\cdots+q_{m}=p$. Then,

$$
\mathbb{E}\left(X_{1}+\cdots+X_{n}\right)^{p}=\sum_{m=1}^{p} \sum_{q \in Q_{m}} \frac{p !}{q_{1} ! \cdots q_{m} !} \frac{n !}{\alpha(q) \cdot(n-m) !} \mu_{q_{1}} \cdots \mu_{q_{m}},
$$


where $\alpha(q)=l_{1} ! \cdots l_{h}$ ! for $q=\left\{q_{1}, \ldots, q_{m}\right\}$ with exactly $h$ distinct terms such that there are $l_{1}$ terms of type $1, l_{2}$ terms of type 2 , etc., so $l_{1}+\cdots+l_{h}=m$ (e.g., for $q=$ $\{2,2,2,3,4,4\} \in Q_{6}$, we have $h=3, l_{1}=3, l_{2}=1$, and $\left.l_{3}=2\right)$. The factor $\frac{n !}{\alpha(q) \cdot(n-m) !}$ arises, because given a multiset $q \in Q_{m}$, there are exactly

$$
\begin{aligned}
\left(\begin{array}{c}
n \\
l_{1}
\end{array}\right)\left(\begin{array}{c}
n-l_{1} \\
l_{2}
\end{array}\right) & \left(\begin{array}{c}
n-l_{1}-l_{2} \\
l_{3}
\end{array}\right) \ldots\left(\begin{array}{c}
n-l_{1}-\cdots-l_{h-1} \\
l_{h}
\end{array}\right) \\
& =\frac{n !}{l_{1} ! \cdot \ldots \cdot l_{h} ! \cdot\left(n-l_{1}-\cdots-l_{h}\right) !}=\frac{n !}{\alpha(q) \cdot(n-m) !}
\end{aligned}
$$

many nonnegative integer-valued sequences $\left(p_{1}, \ldots, p_{n}\right)$ such that $\mu_{p_{1}} \cdots \mu_{p_{n}}=$ $\mu_{q_{1}} \cdots \mu_{q_{m}}$ (equivalently, $\left\{p_{1}, \ldots, p_{n}\right\}=\left\{q_{1}, \ldots, q_{m}, 0\right\}$, as sets).

We have obtained

$$
b_{n}=\mathbb{E}\left(\frac{X_{1}+\cdots+X_{n}}{n}\right)^{p}=\sum_{m=1}^{p} \frac{n !}{n^{p}(n-m) !} \sum_{q \in Q_{m}} \beta(q) \mu(q),
$$

where $\beta(q)=\frac{p !}{\alpha(q) \cdot q_{1} ! \cdots q_{m} !}$ and $\mu(q)=\mu_{q_{1}} \cdots \mu_{q_{m}}$. By homogeneity, we can assume that $\mu_{1}=\mathbb{E} X_{1}=1$. Note that when $X_{1}$ is constant, we get from (5) that

$$
1=\sum_{m=1}^{p} \frac{n !}{n^{p}(n-m) !} \sum_{q \in Q_{m}} \beta(q) .
$$

Because $Q_{p}$ has only one element, namely $\{1, \ldots, 1\}$ and $\mu(\{1, \ldots, 1\})=1$, when we subtract the two equations, the terms corresponding to $m=p$ cancel and we get

$$
b_{n}-1=\sum_{m=1}^{p-1} \frac{n !}{n^{p}(n-m) !} \sum_{q \in Q_{m}} \beta(q)(\mu(q)-1) .
$$

By the monotonicity of moments, $\mu(q) \geq 1$ for every $q$, so $\left(b_{n}\right)$ is a sum of the constant sequence $(1,1, \ldots)$ and the sequences $\left(u_{n}^{(m)}\right)=\left(\frac{n !}{n^{p}(n-m) !}\right), m=1, \ldots, p-1$, multiplied, respectively, by the nonnegative factors $\sum_{q \in Q_{m}} \beta(q)(\mu(q)-1)$. Because sums of log-convex sequences are log-convex, it remains to verify that for each $1 \leq m \leq$ $p-1$, we have $\left(u_{n}^{(m)}\right)^{2} \leq u_{n-1}^{(m)} u_{n+1}^{(m)}, n \geq p^{2}$. The following lemma finishes the proof.

Lemma 7 Let $p \geq 2,1 \leq m \leq p-1$, be integers. Then, the function

$$
f(x)=\log \frac{x(x-1) \cdots(x-m+1)}{x^{p}}
$$

is convex on $\left[p^{2}-1, \infty\right)$.

Proof The statement is clear for $m=1$. Let $2 \leq m \leq p-1$ and $p \geq 3$. We have

$$
x^{2} f^{\prime \prime}(x)=p-1-x^{2} \sum_{k=1}^{m-1} \frac{1}{(x-k)^{2}} .
$$


To see that this is positive for every $x \geq p^{2}-1$ and $2 \leq m \leq p-1$, it suffices to consider $m=p-1$ and $x=p^{2}-1$ (writing $\frac{x}{x-k}=1+\frac{k}{x-k}$, we see that the right-hand side is increasing in $x$ ). Because

$$
\sum_{k=1}^{p-2} \frac{1}{\left(p^{2}-1-k\right)^{2}}=\sum_{k=p^{2}-p+1}^{p^{2}-2} \frac{1}{k^{2}} \leq \sum_{k=p^{2}-p+1}^{p^{2}-2}\left(\frac{1}{k-1}-\frac{1}{k}\right)=\frac{1}{p^{2}-p}-\frac{1}{p^{2}-2},
$$

we have

$$
x^{2} f^{\prime \prime}(x) \geq p-1-\left(p^{2}-1\right)^{2}\left(\frac{1}{p^{2}-p}-\frac{1}{p^{2}-2}\right)=\frac{(p-1)(p+2)}{p\left(p^{2}-2\right)},
$$

which is clearly positive.

\section{Final remarks}

Remark 8 Using majorization-type arguments (see, e.g., [9]), Conjecture 1 can be verified in a rather standard but lengthy way for every $p>1$ and $n=2$. The idea is to establish a pointwise inequality: we conjecture that for nonnegative numbers $x_{1}, \ldots, x_{2 n}$ and a convex function $\phi:[0, \infty) \rightarrow[0, \infty)$, we have

$$
\frac{1}{\left(\begin{array}{c}
2 n \\
n
\end{array}\right)} \sum_{|I|=n} \phi\left(\frac{x_{I} x_{I^{c}}}{n^{2}}\right) \leq \frac{1}{\left(\begin{array}{c}
2 n \\
n+1
\end{array}\right)} \sum_{|I|=n+1} \phi\left(\frac{x_{I} x_{I^{c}}}{n^{2}-1}\right),
$$

where for a subset $I$ of the set $\{1, \ldots, 2 n\}$, we denote $x_{I}=\sum_{i \in I} x_{i}$. We checked that this holds for $n=2$. Taking the expectation on both sides for $\phi(x)=x^{p}$ gives the desired result that $b_{n}^{2} \leq b_{n-1} b_{n+1}$.

Remark 9 It is tempting to ask for generalizations of Conjecture 1 beyond the power functions, say to ask whether the sequence $\left(a_{n}\right)$ defined in (1) is log-convex for every convex function $f$. This is false, as can be seen by taking the function $f$ of the form $f(x)=\max \{x-a, 0\}$ and the $X_{i}$ to be i.i.d. Bernoulli random variables.

Acknowledgment We would like to thank Krzysztof Oleszkiewicz for his great help, valuable correspondence, feedback, and for allowing us to use his slick proof for $p=3$ from Remark 5. We would also like to thank Marta Strzelecka and Michał Strzelecki for the helpful discussions. We are indebted to the referees for their careful reading and useful comments. We are grateful for the excellent working conditions provided by the Summer Undergraduate Research Fellowship 2019 at Carnegie Mellon University during which this work was initiated.

\section{References}

[1] P. Boland, F. Proschan, and Y. Tong, Some majorization inequalities for functions of exchangeable random variables. In: H. W. Block, A. R. Sampson, and T. H. Savits (eds.), Topics in statistical dependence (Somerset, PA, 1987), IMS Lecture Notes Monograph Series, 16, Institute of Mathematical Statistics, Hayward, CA, 1990, pp. 85-91.

[2] S. Boyd and L. Vandenberghe, Convex optimization. Cambridge University Press, Cambridge, MA, 2004. 
[3] F. Brenti, Unimodal, log-concave and Pólya frequency sequences in combinatorics. Mem. Amer. Math. Soc. 81(1989), no. 413, viii + 106 pp.

[4] T. Došlić, Seven (lattice) paths to log-convexity. Acta Appl. Math. 110(2010), no. 3, 1373-1392.

[5] T. Došlić and D. Veljan, Logarithmic behavior of some combinatorial sequences. Discrete Math. 308(2008), no. 11, 2182-2212.

[6] S. Fadnavis, On Brenti's conjecture about the log-concavity of the chromatic polynomial. Euro J. Combin. 33(2012), no. 8, 1842-1846.

[7] W. Feller. An introduction to probability theory and its applications. Vol. II, 2nd ed., John Wiley \& Sons, Inc., New York, London, and Sydney, 1971.

[8] L. Liu and Y. Wang, On the log-convexity of combinatorial sequences. Adv. Appl. Math. 39(2007), no. $4,453-476$.

[9] A. Marshall, I. Olkin, and B. Arnold, Inequalities: theory of majorization and its applications. 2nd ed., Springer Series in Statistics, Springer, New York, 2011.

[10] P. Nayar and K. Oleszkiewicz, Khinchine type inequalities with optimal constants via ultra log-concavity. Positivity 16(2012), no. 2, 359-371.

[11] B. Sagan, Inductive and injective proofs of log concavity results. Discrete Math. 68(1988), nos. 2-3, 281-292.

[12] R. Stanley, Log-concave and unimodal sequences in algebra, combinatorics, and geometry. In: Graph theory and its applications: East and West (Jinan, 1986), Annals of the New York Academy of Sciences, 576, New York Academy of Sciences, New York, 1989, pp. 500-535.

[13] R. Stanley, Positivity problems and conjectures in algebraic combinatorics. In: Mathematics: frontiers and perspectives, American Mathematical Society, Providence, RI, 2000, pp. 295-319.

Department of Mathematical Sciences, Carnegie Mellon University, Pittsburgh, PA 15213, USA.

e-mail: plamkin@andrew.cmu.edu ttkocz@math.cmu.edu 